\title{
Iron Deficiency Anemia in Infants of Hatta Suburb-UAE
}

\author{
Dileep Kumar ${ }^{1 *}$, Zahid Nabi Qureshi ${ }^{1}$ and Mouza Saif Albadwawi, \\ ${ }^{1}$ Specialist Family Physician, Hatta Hospital DHA, United Arab Emirates \\ ${ }^{2}$ Head of Family Medicine Department, Hatta Hospital DHA, United Arab Emirates
}

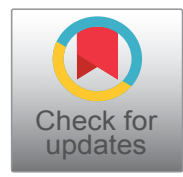

*Corresponding author: Dr. Dileep Kumar, MBBS, FCPS, MRCGP Int, Specialist Family Physician, Hatta Hospital DHA, United Arab Emirates, Tel: +9715-6221-0320, ORCID iD: 0000-0001-8537-1764

\begin{abstract}
Introduction: Worldwide burden of ID estimated to be $43 \%$ globally in 2011 and about $70 \%$ in Central and West Africa and $7-9 \%$ of USA children ages $1-3$ years have iron deficiency (ID). Countries in high caloric nutrition switch have a high prevalence of overweight and obesity, a moderate prevalence of undernutrition and micronutrient deficiencies. Infants and children must obtain $30 \%$ of their daily iron from their diet to provide the necessary iron for new muscle cells and RBCs.

Methods: 259 infants visited child health family medicine clinic Hatta Hospital, UAE for 12 months assessment and vaccination from April 2014 to January 2015. Anemia was defined as per World health organization (hemoglobin $\leq 11$ $\mathrm{gm} / \mathrm{dl}$ )

Results: Anemia was found in 99 infants (38.2\%), Iron deficiency anemia in $60(23.2 \%)$, thalassemia trait in 12 (4.6\%), normocytic anemia in $27(10.4 \%)$ and iron deficiency in $57(22 \%)$. Mild anemia was found 41 infants $(61.3 \%, p$ value 0.000 ) in iron deficiency anemia.

Serum iron 49 infants $(81.7 \%$, p value 0.000$)$, high RDW $52(86.7 \%$, p value 0.000$)$, Mentzer index $54(90 \%$, p value $0.000)$ and Srivastav index $(50 \%, p$ value 0.000$)$ significantly detect iron deficiency anemia.

Conclusion: Iron deficiency anemia (23\%) in this population are consistent with other reports from region but higher than developed countries. Serum iron, high RDW, Mentzer and Srivastav index were significant in detecting iron deficiency anemia in this study. Microcytosis in the study population apart from iron deficiency anemia could be attributed to alpha thalassemia trait in this population.
\end{abstract}

\section{Keywords}

Iron deficiency, Anemia, Infants

\section{Introduction}

Iron deficiency (ID) is the most common micronutrient deficiency in children [1]. Worldwide burden of ID estimated to be $43 \%$ globally in 2011 and about $70 \%$ in Central and West Africa and 7-9\% of USA children ages 1-3 years have ID [2]. ID during fetal development and the first 2 years of life is associated with poor growth and decreases in cognitive, motor, and social emotional development $[3,4]$.

Countries in advanced nutrition switch have a high prevalence of overweight and obesity, a moderate prevalence of undernutrition and micronutrient deficiencies [5]. World Health Organization (WHO) detects deficient and inadequate intakes or status of calcium, iodine, iron, and zinc as well as vitamin A, vitamin D, and folate as commonly reported by many countries in the Middle East region, mostly in children and childbearing age women [6].

A study done by Hopkin, et al., showed the prevalence of anemia was $23 \%$ at 8 months of age, and $18 \%$ at 12 months of age [7]. The prevalence of non-anemic iron deficiency may be as high as $30 \%$ in toddlers from developed countries [8-10].

According to WHO prevalence of anemia severity describes as mild problem $5 \%-19.9 \%$, moderate problem $20 \%-39.9 \%$, and severe problem $\geq 40 \%$; most Arab Middle East countries fall within the category of moderate to severe deficiency $[11,12]$.

IDA was predominant in boys and peak IDA incidence was noted among infants aged 9-12 months. Only $7 \%$ patients exhibited symptoms of IDA, while

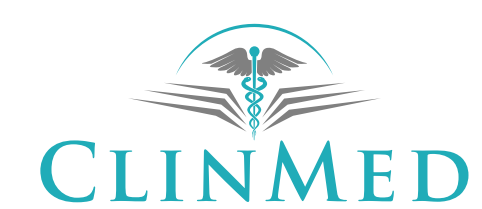

INTERNATIONAL LIBRARY
Citation: Kumar D, Qureshi ZN, Albadwawi MS (2019) Iron Deficiency Anemia in Infants of Hatta Suburb-UAE. Int Arch Nurs Health Care 5:121. doi.org/10.23937/2469-5823/1510121

Accepted: April 17, 2019: Published: April 19, 2019

Copyright: (c) 2019 Kumar D, et al. This is an open-access article distributed under the terms of the Creative Commons Attribution License, which permits unrestricted use, distribution, and reproduction in any medium, provided the original author and source are credited. 
23.6\% patients with severe IDA demonstrated classic symptoms/signs of IDA [13].

Anemia is the most prevalent nutritional disorder among children in the Middle East and North Africa region. The prevalence of anemia in children and adolescents ranges from $11.6 \%$ in Saudi school-aged children to $37 \%-52 \%$ among Egyptian children aged 12 36 months [14-17].

This rise in the prevalence of anemia is attribute to shifting of food consumption patterns and increases in childhood diarrhea [18].

The purpose of this study was to determine the hematological profile, the frequency and severity of iron deficiency anemia (IDA) in one-year old Emeriti infants of Hatta suburb, UAE.

\section{Study Methods}

All infants attended child health family clinic Hatta Hospital-UAE, for one-year assessment and vaccination from April 2014 to January 2015 were included in the study and infants who were not underwent laboratory test were excluded. After history, physical examination conducted by family physician, laboratory test such as complete blood count (CBC), serum ferritin (Fe), serum Iron were advised and follow up appointment given after 4 weeks to review lab test.

Anemia was defined as: Hemoglobin $\leq 11 \mathrm{gm} / \mathrm{dl}$ [19].

Anemia severity classified according to WHO guideline as:

1. Mild anemia: Hemoglobin $10-11 \mathrm{gm} / \mathrm{dl}$

2. Moderate anemia: Hemoglobin 7-9.9 gm/dl

3. Severe anemia: hemoglobin $<7 \mathrm{gm} / \mathrm{dl}$

Iron deficiency anemia defined as; low hemoglobin ( $\leq 11 \mathrm{gm} / \mathrm{dl})$, low MCV ( $\leq 70 \mathrm{fl}$ ) with low serum ferritin (as per laboratory reference, some cases mentioned 4.01 and in other 61$)$ or serum iron $(<43)$, high red cell distribution width (RDW) $\geq 15 \%$.

Thalassemia trait defined as low hemoglobin $(\leq 11$ $\mathrm{gm} / \mathrm{dl}$ ), low $\mathrm{MCV}(\leq 70 \mathrm{fl}$ ) with normal serum ferritin or serum iron, high RBC mass, low RDW $<15 \%$.

Iron deficiency was defined as normal hemoglobin with low serum ferritin or serum iron level.
Three index were used to differentiate Iron Deficiency Anemia with thalassemia trait.

\begin{tabular}{|l|l|l|l|}
\hline & Formula & IDA & $\begin{array}{l}\text { Thalassemia } \\
\text { Trait }\end{array}$ \\
\hline Mentzer index & MCV/RBC & $>13$ & $<13$ \\
\hline RDWI & MCV $\times$ RDW/RBC & $>220$ & $<220$ \\
\hline $\begin{array}{l}\text { Srivastava } \\
\text { index }\end{array}$ & MCH/RBC & $>3.8$ & $<3.8$ \\
\hline
\end{tabular}

All data entered into SPSS software version 24. Mean and Standard Deviation was taken for variables such as Hemoglobin, MCV, RBC mass, MCH, RDW, Serum iron, Serum ferritin and frequency of variables such as anemia, anemia severity, anemia types, Microcytosis, Serum iron, serum ferritin, RDW, RBC mass, Mentzer index, RDWI index, Srivastava index.

Chi-square test was used to differentiate quantitative variables (anemia severity, RBC mass, RDW, Mentzer index, RDWI index, Srivastava index) in Iron deficiency anemia and thalassemia trait. $P$ value of $<0.05$ considered as significant. Prior approval taken from ERC committee and department head of facility.

\section{Results}

Total 320 infants attended child health family clinic Hatta Hospital-UAE, for one-year assessment and vaccination from April 2014 to January 2015, out of these 259 did laboratory test. Mean and Standard Deviation of variables (Hemoglobin, MCV, RBC mass, $\mathrm{MCH}, \mathrm{RDW}$, Serum iron, Serum ferritin) were calculated as shown in Table 1.

Anemia was found in 99 infants (38.2\%), Iron deficiency anemia in 60 (23.2\%), Thalassemia trait in $12(4.6 \%)$ and normocytic anemia in 27 (10.4\%). Iron deficiency was found in 57 infants (22\%). Details of frequency of quantitative variables (anemia, anemia severity, anemia types, Microcytosis, Serum iron, serum ferritin, RDW, RBC mass, Mentzer index, RDWI index, Srivastava index) are shown in Table 2. Details of frequency of anemia variables shown in Table 3 and type of anemia shown in Figure 1.

Among iron deficiency anemia most infants had mild anemia 41 (61.3\%, p value 0.000). Serum iron was significantly low in iron deficiency anemia 49 infants $(81.7 \%, p$ value 0.000$)$ as compare to serum ferritin

Table 1: Mean and standard deviation data.

\begin{tabular}{|l|l|l|l|l|}
\hline Variables & Minimum & Maximum & Mean & Standard Deviation (SD) \\
\hline Hemoglobin level & 7.70 & 14.60 & 11.4402 & 1.08568 \\
\hline MCV value & 50.00 & 84.00 & 69.3977 & 6.61783 \\
\hline RBC mass & 3.90 & 55.00 & 5.2372 & 3.14462 \\
\hline MCH value & 13.50 & 29.20 & 22.6494 & 2.72338 \\
\hline RDW value & 11.70 & 23.40 & 15.6610 & 1.98865 \\
\hline Serum Iron & 10.00 & 137.00 & 53.3923 & 24.03063 \\
\hline Serum Ferritin & 2.20 & 99.00 & 31.3398 & 18.11658 \\
\hline
\end{tabular}


Table 2: Frequency of variables (Total 259).

\begin{tabular}{|c|c|c|}
\hline Variable & No. & Percentage \\
\hline Anemia (Hemoglobin $\leq 11 \mathrm{gm} / \mathrm{dl}$ ) & 99 & $38.2 \%$ \\
\hline \multicolumn{3}{|l|}{ Microcytic Anemia } \\
\hline Iron deficiency anemia (IDA) & 60 & $23.2 \%$ \\
\hline Thalassemia Trait & 12 & $4.6 \%$ \\
\hline Normocytic anemia & 27 & $10.4 \%$ \\
\hline \multicolumn{3}{|l|}{ Anemia severity } \\
\hline Mild (10-11 gm/dl) & 79 & $30.5 \%$ \\
\hline Moderate $(7-9.9 \mathrm{gm} / \mathrm{dl})$ & 20 & $7.7 \%$ \\
\hline \multicolumn{3}{|l|}{ MCV } \\
\hline Microcytosis $(\leq 70 \mathrm{fl})$ & 133 & $51.4 \%$ \\
\hline Normal (> $70 \mathrm{fl})$ & 126 & $48.6 \%$ \\
\hline \multicolumn{3}{|l|}{ Serum Iron } \\
\hline Low (< 43 ug/dl) & 103 & $39.8 \%$ \\
\hline Normal/high (> 43 ug/dl) & 156 & $60.2 \%$ \\
\hline \multicolumn{3}{|l|}{ Serum Ferritin } \\
\hline Low $(<61 \mathrm{ng} / \mathrm{ml})$ & 109 & $42 \%$ \\
\hline Normal/high (> 61 ng/ml) & 150 & $58 \%$ \\
\hline \multicolumn{3}{|l|}{ RBC mass } \\
\hline High ( $\left.\geq 510^{6} / u l\right)$ & 153 & $59 \%$ \\
\hline $\operatorname{Low}\left(<510^{6} / \mathrm{ul}\right)$ & 106 & $41 \%$ \\
\hline \multicolumn{3}{|l|}{ Red cell Distribution Width (RDW) } \\
\hline High ( $\geq 15 \%)$ & 159 & $61.4 \%$ \\
\hline Low $(<15 \%)$ & 100 & $38.6 \%$ \\
\hline Iron deficiency & 57 & $22 \%$ \\
\hline \multicolumn{3}{|l|}{ Mentzer Index (MI index) } \\
\hline$>13$ & 207 & $79.9 \%$ \\
\hline$<13$ & 52 & $20.1 \%$ \\
\hline \multicolumn{3}{|l|}{ Srivastav Index (SI index) } \\
\hline$>3.8$ & 202 & $78 \%$ \\
\hline$<3.8$ & 57 & $22 \%$ \\
\hline \multicolumn{3}{|l|}{ RDWI Index } \\
\hline$>220$ & 116 & $45 \%$ \\
\hline$<220$ & 143 & $55 \%$ \\
\hline
\end{tabular}

Table 3: Frequency of variables in anemia.

\begin{tabular}{|c|c|c|c|}
\hline \multirow[t]{2}{*}{ Variables } & \multicolumn{3}{|c|}{ Anemia (99 cases) } \\
\hline & No. & $\%$ & $P$ value \\
\hline $\begin{array}{l}\text { MCV } \\
\text { Microcytosis }(\leq 70 \mathrm{fl}) \\
\text { Normal }(>70 \mathrm{fl})\end{array}$ & $\begin{array}{l}72 \\
27\end{array}$ & $\begin{array}{l}72.7 \% \\
29.3 \%\end{array}$ & 0.000 \\
\hline $\begin{array}{l}\text { Anemia severity } \\
\text { Mild }(10-11 \mathrm{gm} / \mathrm{dl}) \\
\text { Moderate }(7-9.9 \mathrm{gm} / \mathrm{dl})\end{array}$ & $\begin{array}{l}79 \\
20\end{array}$ & $\begin{array}{l}79.8 \% \\
20.2 \%\end{array}$ & 0.000 \\
\hline $\begin{array}{l}\text { Anemia types } \\
\text { Iron deficiency anemia (IDA) } \\
\text { Thalassemia Trait } \\
\text { Normocytic anemia }\end{array}$ & $\begin{array}{l}60 \\
12 \\
27\end{array}$ & $\begin{array}{l}60.6 \% \\
12.1 \% \\
27.3 \%\end{array}$ & 0.000 \\
\hline $\begin{array}{l}\text { Serum Iron } \\
\text { Low (< } 43 \mathrm{ug} / \mathrm{dl}) \\
\text { Normal/high (> } 43 \mathrm{ug} / \mathrm{dl})\end{array}$ & $\begin{array}{l}63 \\
36\end{array}$ & $\begin{array}{l}63.6 \% \\
36.4 \%\end{array}$ & 0.000 \\
\hline $\begin{array}{l}\text { Serum Ferritin } \\
\text { Low }(<61 \mathrm{ng} / \mathrm{ml}) \\
\text { Normal/high }(>61 \mathrm{ng} / \mathrm{ml})\end{array}$ & $\begin{array}{l}47 \\
52\end{array}$ & $\begin{array}{l}47.5 \% \\
52.5 \%\end{array}$ & 0.196 \\
\hline $\begin{array}{l}\text { RBC mass } \\
\text { High }\left(\geq 510^{6} / \text { ul }\right) \\
\text { Low }\left(<510^{6} / \text { ul }\right)\end{array}$ & $\begin{array}{l}51 \\
48\end{array}$ & $\begin{array}{l}51.5 \% \\
48.5 \%\end{array}$ & 0.068 \\
\hline $\begin{array}{l}\text { Red cell Distribution Width } \\
\text { (RDW) } \\
\text { High }(\geq 15 \%) \\
\text { Low }(<15 \%)\end{array}$ & $\begin{array}{l}73 \\
26\end{array}$ & $\begin{array}{l}73.7 \% \\
26.3 \%\end{array}$ & 0.002 \\
\hline $\begin{array}{l}\text { Mentzer Index (MI index) } \\
>13 \\
<13\end{array}$ & $\begin{array}{l}92 \\
7\end{array}$ & $\begin{array}{l}93 \% \\
7 \%\end{array}$ & 0.000 \\
\hline $\begin{array}{l}\text { Srivastav Index (SI index) } \\
>3.8 \\
<3.8\end{array}$ & $\begin{array}{l}62 \\
37\end{array}$ & $\begin{array}{l}62.6 \% \\
37.4 \%\end{array}$ & 0.000 \\
\hline $\begin{array}{l}\text { RDWI Index } \\
>220 \\
<220\end{array}$ & $\begin{array}{l}53 \\
46\end{array}$ & $\begin{array}{l}53.5 \% \\
46.5 \%\end{array}$ & 0.026 \\
\hline
\end{tabular}

\section{ANEMIA TYPES}

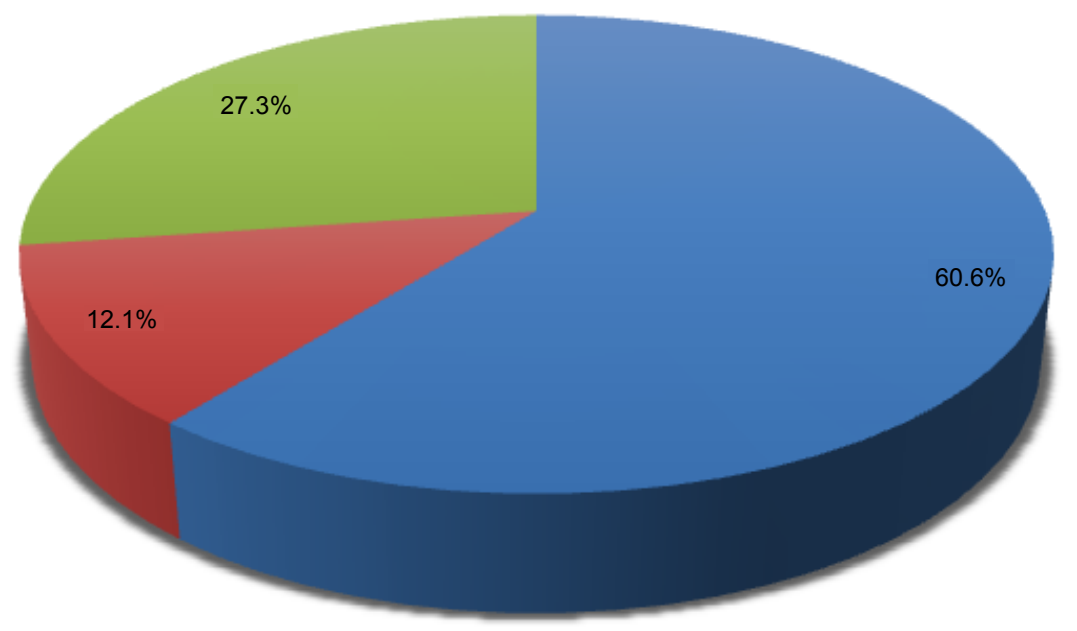

Iron deficiency anemia $\quad$ Thalassemia trait $\quad$ Normocytic anemia

Figure 1: Anemia types. 
Table 4: Frequency of variables in iron deficiency anemia.

\begin{tabular}{|c|c|c|c|}
\hline \multirow[t]{2}{*}{ Variables } & \multicolumn{3}{|c|}{$\begin{array}{l}\text { Iron deficiency Anemia } \\
\text { (60 infants) }\end{array}$} \\
\hline & No. & $\%$ & $P$ value \\
\hline $\begin{array}{l}\text { Anemia severity } \\
\text { Mild }(10-11 \mathrm{gm} / \mathrm{dl}) \\
\text { Moderate }(7-9.9 \mathrm{gm} / \mathrm{dl})\end{array}$ & $\begin{array}{l}41 \\
19\end{array}$ & $\begin{array}{l}68.3 \% \\
31.7 \%\end{array}$ & 0.000 \\
\hline $\begin{array}{l}\text { Serum Iron } \\
\text { Low (< } 43 \text { ug/dl) } \\
\text { Normal/high (> } 43 \text { ug/dl) }\end{array}$ & $\begin{array}{l}49 \\
11\end{array}$ & $\begin{array}{l}81.7 \% \\
18.3 \%\end{array}$ & 0.000 \\
\hline $\begin{array}{l}\text { Serum Ferritin } \\
\text { Low }(<61 \mathrm{ng} / \mathrm{ml}) \\
\text { Normal/high }(>61 \mathrm{ng} / \mathrm{ml})\end{array}$ & $\begin{array}{l}33 \\
27\end{array}$ & $\begin{array}{l}55 \% \\
45 \%\end{array}$ & 0.025 \\
\hline $\begin{array}{l}\text { RBC mass } \\
\text { High }\left(\geq 510^{6} / \mathrm{ul}\right) \\
\text { Low }\left(<510^{6} / \mathrm{ul}\right)\end{array}$ & $\begin{array}{l}41 \\
19\end{array}$ & $\begin{array}{l}68.3 \% \\
31.7 \%\end{array}$ & 0.102 \\
\hline $\begin{array}{l}\text { Red cell Distribution Width } \\
\text { (RDW) } \\
\text { High }(\geq 15 \%) \\
\text { Low }(<15 \%)\end{array}$ & $\begin{array}{l}52 \\
08\end{array}$ & $\begin{array}{l}86.7 \% \\
13.3 \%\end{array}$ & 0.000 \\
\hline $\begin{array}{l}\text { Mentzer Index (MI index) } \\
>13 \\
<13\end{array}$ & $\begin{array}{l}54 \\
6\end{array}$ & $\begin{array}{l}90 \% \\
10 \%\end{array}$ & 0.000 \\
\hline $\begin{array}{l}\text { Srivastav Index (SI index) } \\
>3.8 \\
<3.8\end{array}$ & $\begin{array}{l}30 \\
30\end{array}$ & $\begin{array}{l}50 \% \\
50 \%\end{array}$ & 0.000 \\
\hline $\begin{array}{l}\text { RDWI Index } \\
>220 \\
<220\end{array}$ & $\begin{array}{l}22 \\
38\end{array}$ & $\begin{array}{l}36.7 \% \\
63.3 \%\end{array}$ & 0.183 \\
\hline
\end{tabular}

33 (55\%, p value 0.025$)$. RDW was significantly high in 52 (86.7\%, $p$ value 0.000). Among indexes, Mentzer and Srivastav index were significant in detecting iron deficiency anemia 54 (90\%, p value 0.000 ) and 30 infants $(50 \%$, p value 0.000$)$ respectively, while RDWI index 22 infants (36.7\%, $p$ value 0.183 ) as shown in Table 4.

Srivastav index significantly detect thalassemia trait $30(50 \%, p$ value 0.000$)$ while Mentzer index and RDWI index were not significant in our study. Details of variables among thalassemia trait shown in Table 5.

\section{Discussion}

Infants and children must obtain $30 \%$ of their daily iron from their diet to provide the necessary iron for new muscle cells and RBCs. Both the United States Department of Health and Human Services and the World Health Organization (WHO) have set goals to reduce ID and iron deficiency anemia (IDA). The WHO priorities have now been adopted as Priority Nutrition Indicators for the United Nation's post-2015 Sustainable Development Goals [20-22].

The principal risk factors associated with IDA were age 6-18 months, male sex, higher birth order, consumption of cow's milk, predominant breastfeeding $>6$ months, and/or low iron intake.
Table 5: Frequency of variables in thalassemia trait.

\begin{tabular}{|c|c|c|c|}
\hline \multirow[t]{2}{*}{ Variables } & \multicolumn{3}{|c|}{$\begin{array}{l}\text { Thalassemia trait } \\
\text { (12 infants) }\end{array}$} \\
\hline & No. & $\%$ & $P$ value \\
\hline $\begin{array}{l}\text { Anemia severity } \\
\text { Mild }(10-11 \mathrm{gm} / \mathrm{dl}) \\
\text { Moderate }(7-9.9 \mathrm{gm} / \mathrm{dl})\end{array}$ & $\begin{array}{l}12 \\
00\end{array}$ & $\begin{array}{l}100 \% \\
0 \%\end{array}$ & 0.000 \\
\hline $\begin{array}{l}\text { RBC mass } \\
\text { High }(\geq 5) \\
\text { Low }(<5)\end{array}$ & $\begin{array}{l}08 \\
04\end{array}$ & $\begin{array}{l}66.7 \% \\
33.3 \%\end{array}$ & 0.766 \\
\hline $\begin{array}{l}\text { Red cell Distribution Width } \\
\text { (RDW) } \\
\text { High }(\geq 15) \\
\text { Low }(<15)\end{array}$ & $\begin{array}{l}11 \\
01\end{array}$ & $\begin{array}{l}91.7 \% \\
08.3 \%\end{array}$ & 0.032 \\
\hline $\begin{array}{l}\text { Mentzer Index (MI index) } \\
>13 \\
<13\end{array}$ & $\begin{array}{l}11 \\
01\end{array}$ & $\begin{array}{l}91.7 \% \\
08.3 \%\end{array}$ & 0.469 \\
\hline $\begin{array}{l}\text { Srivastav Index (SI index) } \\
>3.8 \\
<3.8\end{array}$ & $\begin{array}{l}05 \\
07\end{array}$ & $\begin{array}{l}41.7 \% \\
58.3 \%\end{array}$ & 0.006 \\
\hline $\begin{array}{l}\text { RDWI Index } \\
>220 \\
<220\end{array}$ & $\begin{array}{l}04 \\
08\end{array}$ & $\begin{array}{l}33.3 \% \\
66.7 \%\end{array}$ & 0.556 \\
\hline
\end{tabular}

Routine screening for ID and IDA between 6-24 months is recommended, especially for children living in areas with a high prevalence of ID. In upper-income countries, a full blood count is obtained, which will give hemoglobin, hematocrit, mean corpuscular volume, and RBC distribution width. In children with IDA, mean corpuscular volume will be decreased and RBC distribution width will most likely be increased. In some settings, ferritin is also measured as part of the screening process. However, ferritin values can be misleading in children living in areas where the infectious disease burden is high because ferritin is also an acute-phase protein.

It is recommended that full-term breastfed infants should start an iron supplement at 4 months (elemental iron $1 \mathrm{mg} / \mathrm{kg}$ daily, maximum $15 \mathrm{mg}$ ) and the supplement should be continued until the infant is taking sufficient quantities of iron-rich complementary foods [1].

Full-term and preterm babies should be taking complementary foods by 6 months of age. Iron-rich complementary foods include meat (lamb, chicken, beef, and pork), baby cereals (including fortified rice), and some vegetables (green beans, peas, and spinach) $[23,24]$.

\section{Conclusion}

- Iron deficiency anemia (23\%) in this population are consistent with other reports from region but higher than developed countries.

- The microcytosis in the study population apart from anemia could be attributed to alpha thalassemia trait and a normal variant microcytosis which is highly 
prevalent in the study population.

- Iron deficiency was found in $22 \%$ infants, warrant early interventions to reduce the risk of developing future anemia.

- Need to conduct studies with large sample size, which will help and signify the magnitude of the IDA.

\section{References}

1. Kassebaum NJ, Jasrasaria R, Naghavi M, Wulf SK, Johns $\mathrm{N}$, et al. (2014) A systematic analysis of global anemia burden from 1990 to 2010. Blood 123: 615-624.

2. Engle-Stone R, Aaron GJ, Huang J, Wirth JP, Namaste SM, et al. (2017) Predictors of anemia in preschool children: Biomarkers Reflecting Inflammation and Nutritional Determinants of Anemia (BRINDA) project. Am J Clin Nutr 106: 402S-415S.

3. Clark KM, Li M, Zhu B, Liang F, Shao J, et al. (2017) Breastfeeding, mixed, or formula feeding at 9 months of age and the prevalence of iron deficiency and iron deficiency anemia in two cohorts of infants in China. J Pediatr 181: 56-61.

4. Geng F, Mai X, Zhan J, Xu L, Zhao Z, et al. (2015) Impact of fetal-neonatal iron deficiency on recognition memory at 2 months of age. J Pediatr 167: 1226-1232.

5. Regional strategy on nutrition 2010-2019 and Plan of action. World Health Organization.

6. Regional Committee for the Eastern Mediterranean (2010) Regional strategy on nutrition 2010-2019. World Health Organization.

7. Hopkins D, Emmett P, Steer C, Rogers. I, Noble S, et al. (2007) Infant feeding in the second 6 months of life related to iron status: An observational study. Arch Dis Child 92: 850-854.

8. Looker AC, Dallman PR, Carroll MD, Gunter EW, Johnson CL (1997) Prevalence of iron deficiency in the United States. JAMA 277: 973-976.

9. Soh P, Ferguson EL, McKenzie JE, Homs MY, Gibson RS (2004) Iron deficiency and risk factors for lower iron stores in 6-24-month-old New Zealanders. Eur J Clin Nutr 58: 7179.

10. Grant CC, Wall CR, Brunt D, Crengle S, Scragg R (2007) Population prevalence and risk factors for iron deficiency in Auckland, New Zealand. J Pediatr Child Health 43: 532538.

11. Mhanna RG, Rahal M, Iskandarani M, Hammoudi D (2016) Incidence and risk factors associated with iron deficiency anemia among hospitalized Lebanese infants. Int J Pharm Pract 24: 203-208.

12. The Global Prevalence of Anemia in 2011. World Health Organization.

13. Eun Young Joo, Keun Young Kim, Dong Hyun Kim, Ji-Eun Lee, Soon Ki Kim (2016) Iron deficiency anemia in infants and toddlers. Blood Res 51: 268-273.

14. Austin AM, Fawzi W, Hill AG (2012) Anemia among Egyptian Children between 2000 and 2005: Trends and predictors. Matern Child Nutr 8: 522-532.

15. Abou-Zeid AH, Abdel-Fattah MM, Al-Shehri AS, Hifnawy TM, Al-Hassan SA (2006) Anemia and nutritional status of schoolchildren living at Saudi high altitude area. Saudi Med J 27: 862-869.

16. Tawfik AA, Hanna ET, Abdel-Maksoud AM (2015) Anemia and iron deficiency anemia in Egypt. IOSR Journal of Pharmacy 5: 30-34.

17. Elalfy MS, Hamdy AM, Maksoud SS, Megeed RI (2012) Pattern of milk feeding and family size as risk factors for iron deficiency anemia among poor Egyptian infants 6 to 24 months old. Nutr Res 32: 93-99.

18. Ministry of Health and Population, El-Zanaty and Associates, DHS Program ICF International (2014) Egypt demographic and health survey 2014: Main findings.

19. (2011) Hemoglobin concentrations for the diagnosis of anemia and assessment of severity. Vitamin and Mineral Nutrition Information System. World Health Organization, Geneva, Switzerland.

20. (2011) Serum ferritin concentrations for the assessment of iron status and iron deficiency in populations. Vitamin and Mineral Nutrition Information System. World Health Organization, Geneva, Switzerland.

21. Bates-Eamer N, Carin B, Lee MH, Lim W, Kapila M (2015) Post-2015 Development agenda: Goals, targets and indicators - Special Report.

22. United Nations Standing Committee on Nutrition (2014) Nutrition and the Post-2015 sustainable development goals - A technical note.

23. Agostoni C, Decsi T, Fewtrell M, Goulet O, Kolacek S, et al. (2008) Complementary feeding: A commentary by the ESPGHAN Committee on Nutrition. J Pediatr Gastroenterol Nutr 46: 99-110.

24. Gupta S, Agarwal R, Aggarwal KC, Chellani H, Duggal A, et al. (2017) Complementary feeding at 4 versus 6 months of age for preterm infants born at less than 34 weeks of gestation: A randomized, open-label, multicenter trial. Lancet Glob Health 5: e501-e511. 